\title{
Risk factors for renal failure: The WHO multinational study of vascular disease in diabetes
}

\author{
H.M. Colhoun ${ }^{1}$, E.T.Lee ${ }^{2}$, P.H. Bennett ${ }^{3}$, M.Lu ${ }^{2}$, H.Keen ${ }^{4}$, S-LWang ${ }^{1}$, L. K. Stevens ${ }^{1}$, J.H. Fuller ${ }^{1}$ \\ and the WHO Multinational Study Group ${ }^{5}$ \\ ${ }^{1}$ EURODIAB, Department of Epidemiology and Public Health, University College London, London, UK \\ ${ }^{2}$ Centre for American Indian Health Research and Department of Biostatistics and Epidemiology, College of Public Health, \\ University of Oklahoma Health Sciences Centre, Oklahoma City, USA \\ ${ }^{3}$ Phoenix Epidemiology and Clinical Research Branch, NIDDK, Phoenix, Arizona, USA \\ ${ }^{4}$ Unit for Metabolic Medicine, Guy's Hospital, London, UK \\ ${ }^{5}$ See reference 45 for names and affiliations of Investigators
}

\section{Abstract}

Aims/hypothesis. We aimed to examine risk factors for, and differences in, renal failure in diabetic patients from 10 centres.

Methods. Risk factors for renal failure were examined in 3558 diabetic patients who did not have renal disease at baseline in the WHO Multinational Study of Vascular Disease in Diabetes (WHO MSVDD). Results. In 959 subjects with Type I (insulin-dependent) diabetes mellitus and 2559 with Type II (noninsulin-dependent) diabetes mellitus, the average follow-up was 8.4 years $( \pm 2.7)$. By the end of the follow-up period 53 patients in the Type I diabetic group and 134 patients in the Type II diabetic group had developed renal failure (incidence rate 6.3:1000 person years). Increasing age and duration of diabetes were associated with renal failure in Type II and Type I diabetes. In Type II diabetes duration of diabetes was a more important risk factor than age. In both Type I and Type II diabetic retinopathy and proteinuria were strongly associated with renal failure. Systolic blood pressure was associated with renal failure in Type I but not in Type II diabetic patients. ECG ab- normalities at baseline, self-reported smoking and cholesterol were not associated with renal failure. Triglycerides were measured in a subset of centres. Among those with Type II, but not Type I diabetes, triglycerides were associated with renal failure independently of systolic blood pressure, proteinuria or retinopathy. In Type II diabetes fasting plasma glucose was associated with renal failure independently of other risk factors.

Conclusion/interpretation. We have confirmed the role of proteinuria and retinopathy as markers of renal failure and the importance of hyperglycaemia in renal failure in Type I and Type II diabetes. Plasma triglycerides seem to be an important predictor of renal failure in Type II diabetes. In Type I diabetes systolic blood pressure is an important predictor of renal failure. [Diabetologia (2001) 44 [Suppl 2]: S 46-S 53]

Keywords Renal failure, nephropathy, Type I (insulin-dependent) diabetes mellitus, Type II (non-insulin-dependent) diabetes mellitus, triglycerides, systolic blood pressure, plasma glucose, smoking, cholesterol, retinopathy, ECG.
Diabetes accounts for one third or more of all new end stage renal disease in the United States and accounts for at least $16 \%$ of all new patients going

Corresponding author: Dr H.M. Colhoun, EURODIAB, Department of Epidemiology and Public Health, University College London, 1-19 Torrington Place, London WC1E 6BT, UK Abbreviations: WHO MSVDD, World Health Organization Multinational Study of Vascular Disease in Diabetes; RR, relative risk; SRR, standardised estimate of the relative risk; SBP, systolic blood pressure; DBP, diastolic blood pressure. onto renal replacement therapy in Europe [1]. The most common cause of end stage renal disease in diabetes is diabetic glomerulosclerosis (nephropathy).

Previous studies have shown that hyperglycaemia is an important risk factor for diabetic renal disease $[2,3]$. More controversy exists on the extent to which systolic blood pressure and lipid disturbances are risk factors for renal disease rather than being a consequence of it. Data on risk factors for renal disease in prospective studies of diabetes are sparse. The 
WHO Multinational Study of Vascular Disease in Diabetes (WHO MSVDD) is a prospective study designed to examine the frequency of diabetic complications and identify risk factors for their development and outcome in Type I (insulin-dependent) and Type II (non-insulin dependent) diabetes mellitus. The study started in 1975 and follow-up of deaths ended in 1988. With over 43670 person years of follow-up it is in a unique position to provide information on risk factors for renal failure. Another paper in this supplement focuses on risk factors for and prevalence of albuminuria, an early marker of renal failure, for which only prevalence data rather than incidence data were available [4].

\section{Subjects and methods}

Methods of patient selection and examination for the WHO MSVDD have been described in the first paper in this series [5] and in detail previously [6, 7]. The risk factors for renal failure included in this analysis were cigarette smoking, blood pressure, serum cholesterol and triglyceride, plasma glucose, proteinuria, retinopathy and ECG abnormalities. The methods of assessing these factors are described elsewhere [5]. For the present analysis categories of retinopathy have been combined into two: any retinopathy or none. Three degrees of proteinuria (heavy, light or none) were defined by the salicylsulphonic acid test, in which the degree of turbidity caused by precipitation of protein was assessed visually and classified as light to heavy on a single spot-urine sample $[6,7]$. Light proteinuria was considered present if there was slight turbidity on precipitation assessed visually. Heavy proteinuria was considered present if there was a distinct or heavy turbidity or a flocculent precipitate. If there was no turbidity then proteinuria was considered absent. For between-centre comparisons patients were grouped according to four geographical regions, i.e. European (London, Switzerland, Berlin, Warsaw and Zagreb); East Asian (Hong Kong and Tokyo); Cuban (Havana) and American Indian (Oklahoma and Arizona).

By the $1^{\text {st }}$ of January 1988 vital status had been ascertained for at least $95 \%$ of the original participants. Those who were alive had a second examination at which whether the patient had renal failure or was receiving renal support (dialysis or transplantation) was recorded. For those alive but not examined, medical records were reviewed for a history of renal failure [5].

For this analysis renal failure was defined as receiving renal support or having a history of renal failure at morbidity followup, or death where ICD-9 codes 580-589 (nephritis, nephrosis and nephrotic syndrome) or 250.3 (diabetic nephropathy) were mentioned anywhere on the final categorisation of cause of death (i.e. either as underlying or contributory cause of death).

Statistical analysis. To examine the association between renal failure and risk factors age standardised incidence rates (per 1000 person years) were calculated by each category of risk factor, where the rates were directly standardised to the age distribution of the whole sample. Those with heavy proteinuria at baseline were considered to have renal disease and were therefore excluded from this analysis of the incidence of renal failure.

Using the Cox proportional hazard model, age adjusted relative risks (RRs) were also calculated for each category. The relative risk was calculated as the exponent of the beta coefficient of the risk factor in the Cox proportional hazards model, where the model contained the risk factor as a categorical variable and age as a covariate. For continuous variables, initially the distribution was split into categories and the RR was the risk of an event associated with having a value in that particular category relative to the risk of an event associated with having a value in the lowest category. For categorical variables the $\mathrm{RR}$ was the risk of an event associated with each category relative to the risk of an event when that risk factor was absent. Any associations were considered statistically significant if the $95 \%$ confidence interval (CI) did not include a value of 1 (i.e. $p<0.05)$. For continuous risk factors the standardised estimates of relative risk (SRRs), adjusted for age, were also calculated. These were the relative risks associated with an increase in 1 standard deviation of the risk factor, again estimated by the Cox proportional hazard model.

To assess the independent association of all risk factors with renal failure, multivariate Cox proportional hazards modeling was carried out. Age, sex and duration of diabetes, were included as covariates in the model and the roles of systolic blood pressure, proteinuria and retinopathy were investigated as risk factors for renal failure.

Because serum triglyceride and plasma glucose were only measured in a sub-sample of 1778 and 2286 patients respectively, the above modeling was repeated first with triglycerides (Model 2) then with fasting plasma glucose (Model 3) included. Serum triglyceride and plasma glucose were log transformed in these analyses because their distributions were skewed. All analyses were done using the SAS statistical package. A $p$ value of less than 0.05 was considered statistically significant.

\section{Results}

There were 4743 participants at baseline in the 10 centres who participated in the morbidity and mortality follow-up. Of these, 516 had either heavy proteinuria or a history of renal failure at baseline and were excluded from this analysis. A further 24 had no morbidity or mortality information. A further 645 participants were known to be alive by close of follow-up but their renal status was not known. These were excluded from the analysis. The risk factor profile (cholesterol, triglycerides, blood pressure) of those excluded was more similar to the group that did not subsequently develop renal failure than those who did.

Thus for this analysis of renal failure 3558 diabetic patients were followed-up (1 705 men and 1853 women), of whom 959 were classified as having Type I diabetes and 2599 as having Type II diabetes. The average follow-up was 8.4 years $( \pm 2.7)$. By the end of follow-up 187 people (53 with Type I, 134 with Type II diabetes) had developed renal failure (Table 1), an incidence rate of 6.3 per 1000 person years. Of these 87 were defined on the basis of a renal disease death and 100 were noted to have renal failure at morbidity follow-up. Of those with renal failure at morbidity follow-up, 47 had died , 30 of whom had renal disease noted as either underlying or contributory cause of death. 
Table 1. Incidence of renal failure by type of diabetes and geographical region

\begin{tabular}{|c|c|c|c|}
\hline & $\begin{array}{l}\text { Type I (insulin-dependent) } \\
\left.\text { diabetes mellitus } n \text { (rate }{ }^{a}\right)\end{array}$ & $\begin{array}{l}\text { Type II (non-insulin-dependent) } \\
\text { diabetes mellitus } n\left(\text { rate }^{\mathrm{a}}\right)\end{array}$ & Total $n$ (rate) \\
\hline Renal failure at morbidity follow-up & $22(2.9)$ & $78(3.5)$ & $100(3.3)$ \\
\hline Renal failure death & $31(3.8)$ & $56(2.1)$ & $87(2.6)$ \\
\hline \multicolumn{4}{|l|}{ Renal failure by geographical region } \\
\hline Europeans & $33(6.1)$ & $31(2.9)$ & $64(4.0)$ \\
\hline East Asians $10(16.7)^{\mathrm{d}}$ & $10(4.7)$ & $20(7.5)$ & \\
\hline
\end{tabular}

a Age adjusted, per thousand person years.

${ }^{\mathrm{b}}$ Renal failure mentioned on death certificate.

${ }^{\mathrm{c}}$ Larger than European rate $p<0.05$.

${ }^{\mathrm{d}}$ Larger than European rate $p<0.01$

The numbers of events and age-adjusted event rates for renal failure by type of diabetes are shown in Table 1. Renal failure rates, adjusted for age, were higher in those with Type I diabetes (7.6:1000 person years) than in those with Type II diabetes (5.9:1000 person years, $p=0.003)$. To assess whether renal failure rates were similar across centres, rates were compared by the four geographical regions. Compared with those in Europe, patients with Type I diabetes from East Asia and Cuba had higher renal failure incidence, adjusted for age (Table 1). Among those with Type II diabetes, Native Americans had a higher incidence of renal failure than those from Europe, adjusted for age (Table 1).

Baseline risk factors and age-adjusted renal failure (Table 2). In Type I diabetes, age (RR for one year $=1.03, p=0.24)$ and duration (RR for one year $=1.02, p=0.21$ ) were similarly predictive of renal failure. In Type II diabetes duration was more strongly predictive of renal failure than age (RR for age $=1.03, \quad p=0.07, \quad \mathrm{RR} \quad$ for duration $=1.09$ $p<0.001)$. Renal failure rates were higher in men than in women both for patients with Type I diabetes (6.2 and 7.9: 1000 person years for men and women respectively) and with Type II diabetes (5.0 and 6.8: 1000 person years for men and women respectively) but these differences were not statistically significant.

For Type I diabetic patients, factors at baseline related to age-adjusted renal failure were systolic blood pressure, the presence of retinopathy and light proteinuria (Table 2). Smoking status, triglycerides, cholesterol, ECG abnormalities and plasma glucose did not show any association with renal failure. There were no differences between sexes in the pattern of these associations.

Among those with Type II diabetes, higher triglycerides and plasma glucose were associated with age adjusted renal failure, whereas systolic blood pressure, total cholesterol, abnormal ECG and smoking were not (Table 2). The presence of retinopathy and light proteinuria at baseline were also predictive of renal failure. There were no differences in the pattern of these associations between the sexes (data not shown). As there was little difference in the univariate associations between sexes, the multivariate analyses were carried out for both sexes combined with adjustment for sex in the model.

The age-adjusted analysis of the association of the factors in Table 2 with renal failure was repeated excluding deaths where renal disease was mentioned on the death certificate but was not the underlying cause of death. The pattern of associations was unchanged except that the magnitude of the association of triglycerides with renal failure increased slightly in those with Type II diabetes.

Baseline risk factors and renal failure-multivariate analysis. Since self-reported smoking, ECG status and cholesterol were not associated with renal failure adjusted for age, duration, and sex, they were not included in the subsequent models. Table 3 shows the relative risks for renal failure estimated from the Cox proportional hazards model including age, duration, and sex as covariates and including systolic blood pressure, with further adjustment for proteinuria and retinopathy (Model 1) and with the inclusion of triglycerides (Model 2) and fasting plasma glucose (Model 3).

In Type I but not Type II diabetic patients, systolic blood pressure was associated with renal failure independent of baseline proteinuria and also of retinopathy. There was no interaction between retinopathy and systolic blood pressure. In Type I and Type II diabetic retinopathy and baseline proteinuria were strongly and independently associated with renal failure. Model 2 contained the same variables as Model 1 with the addition of triglycerides. Since there was no association between triglycerides and renal failure in Type I diabetes this model was only carried out for Type II diabetes. Triglycerides were independently associated with renal failure even after adjusting for proteinuria, retinopathy and systolic blood pressure. There was no interaction between the effect of tri- 
Table 2. Age-adjusted incidence of renal failure and the relative risk (RR) associated with duration of diabetes, systolic blood pressure, serum cholesterol, serum triglyceride, plasma glucose, smoking, proteinuria, retinopathy and ECG abnormalities, by Type I and Type II diabetes

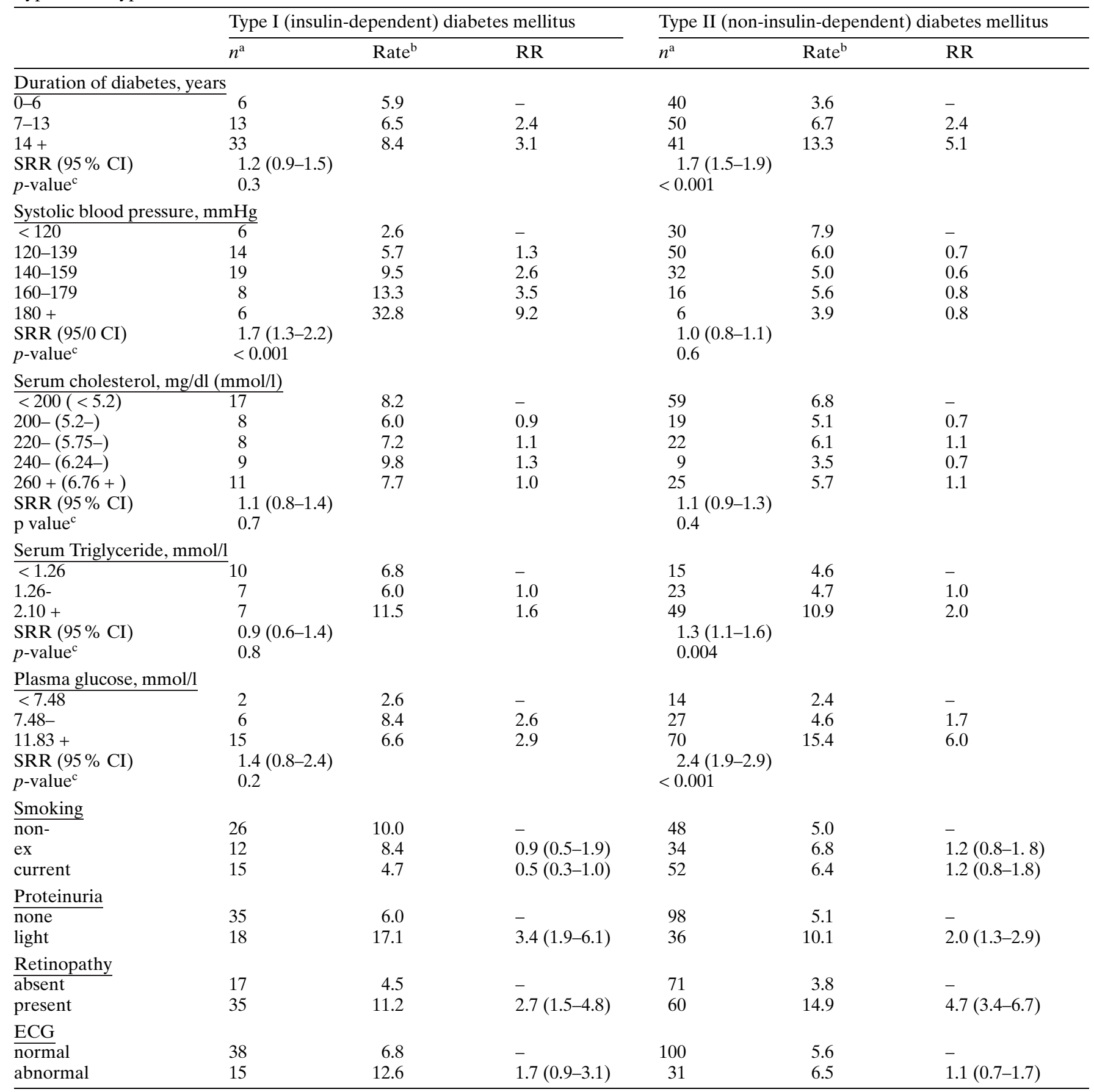

${ }^{\mathrm{a}}$ Number of CVD deaths; ${ }^{\mathrm{b}}$ Age-adjusted rate per 1,000 person years;

${ }^{c} p$ value relates to the significance of the risk factor as a continuous variable

glycerides and retinopathy. Among those with Type II diabetes fasting plasma glucose at baseline strongly predicted renal failure independently of the other factors in the model (Model 3). There was no interaction between fasting plasma glucose and retinopathy.

At baseline $25 \%$ of Type I diabetic patients and $39 \%$ of Type II diabetic patients were on antihyper- tensive therapy. When the age adjusted analysis (Table 2) and the multivariate analysis (Table 3) were carried out using hypertension (defined as a systolic blood pressure $\geq 140 \mathrm{mmHg}$ or a diastolic blood pressure $\geq 90 \mathrm{mmHg}$ or on anti-hypertensive therapy) instead of systolic blood pressure, the conclusions did not alter. For Type I diabetic patients the age adjust- 
Table 3. Cox proportional hazards models for renal failure

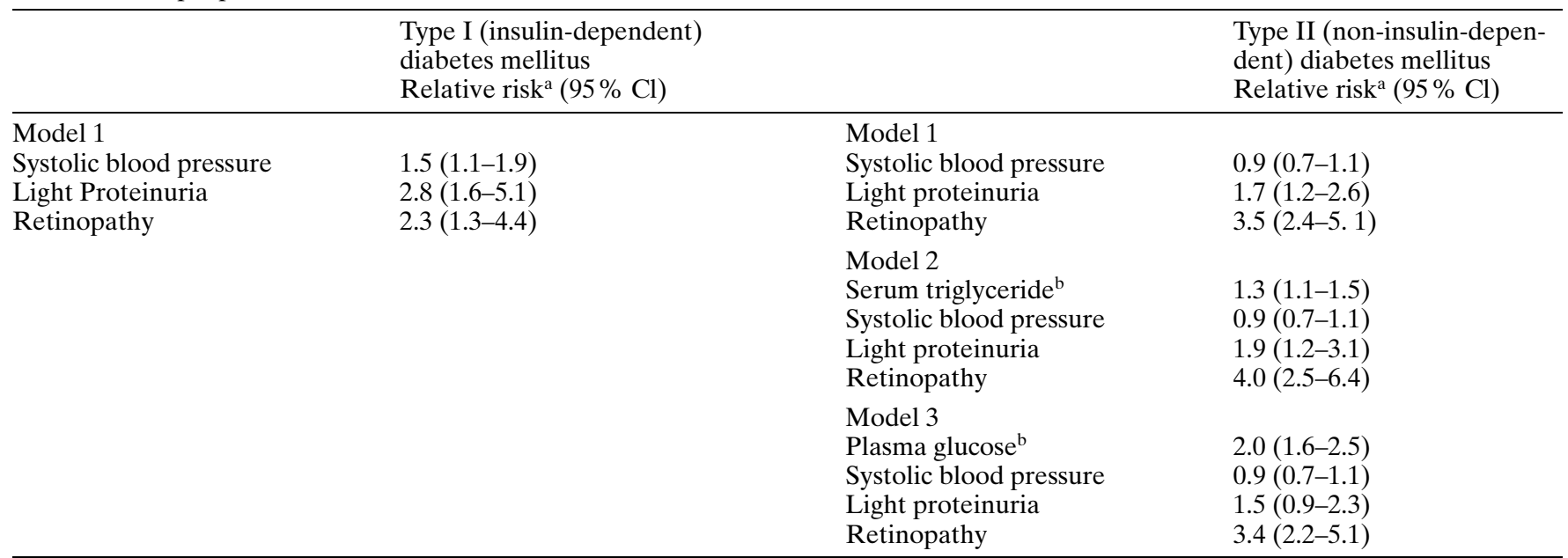

${ }^{a}$ Adjusted for age, duration of diabetes and sex. Standardised relative risks are presented for systolic blood pressure, serum triglyceride and plasma glucose; ${ }^{\mathrm{b}}$ The logged variable was used

Table 4. Incidence of renal failure for Native Americans relative to that of Europeans in patients with Type II (non-insulin-dependent) diabetes mellitus

\begin{tabular}{llll}
\hline Model & $\begin{array}{l}\text { Model 1 }(n=1983) \\
\text { Relative risk }(95 \% \mathrm{Cl})\end{array}$ & $\begin{array}{l}\text { Model 2 }(n=1289) \\
\text { Relative risk }(95 \% \mathrm{Cl})\end{array}$ & $\begin{array}{l}\text { Model } 3(n=1455) \\
\mathrm{Relative} \text { risk }(95 \% \mathrm{Cl})\end{array}$ \\
\hline $\begin{array}{l}\text { Age, duration of diabetes and sex } \\
\text { Age, duration of diabetes, sex, and systolic blood pressure }\end{array}$ & $\begin{array}{l}2.09(1.30,3.35) \\
1.97(1.21,3.19)\end{array}$ & $1.71(0.96,3.03)$ & $1.65(0.92,2.94)$ \\
$\begin{array}{l}\text { Age, duration of diabetes, sex, systolic blood pressure and } \\
\text { retinopathy }\end{array}$ & $1.90(1.11,3.38)$ & $1.79(1.02,3.15)$ \\
$\begin{array}{l}\text { Age, duration of diabetes, sex, systolic blood pressure, } \\
\text { retinopathy and proteinuria }\end{array}$ & $1.94(1.19,3.17)$ & $1.48(0.83,2.64)$ & $1.45(0.82,2.56)$ \\
$\begin{array}{l}\text { Age, duration of diabetes, sex, systolic blood pressure, } \\
\text { retinopathy, proteinuria and triglyceride }\end{array}$ & & $1.52(0.85,2.71)$ & $1.45(0.82,2.57)$ \\
$\begin{array}{l}\text { Age, duration of diabetes, sex, systolic blood pressure, } \\
\text { retinopathy, proteinuria and plasma glucose }\end{array}$ & & & $1.74,2.42)$ \\
\hline
\end{tabular}

ed RR $(95 \%$ CI) was $2.2(1.2,4.0)$ and for Type II diabetic patients it was $1.1(0.8,1.6)$. The multivariate analysis showed that, for Type I diabetic patients, the RR (95\%) for hypertension in Model 1 was 1.8 $(1.0,1.3)$. For Type II diabetic patients the RRs (95\%) for hypertension, in Models 1, 2 and 3 were $1.0(0.7,1.4), 0.9(0.6,1.3)$ and $0.8(0.5,1.3)$, respectively.

Table 4 shows the effect of adjusting for various risk factors on the difference in incidence of renal failure between the Native American group and patients with Type II diabetes from European Centres. Adjusted for diabetes duration, age and sex, Native Americans had more than twice the risk of developing renal failure $(\mathrm{RR}=2.09)$. There was little attenuation of this risk on adjustment for baseline retinopathy, systolic blood pressure or light proteinuria (Table 4, Model 1). In Model 2 and Model 3, although the relative risks associated with Native American ethnicity are similar, because there are fewer subjects in the analysis the association with ethnicity does not reach statistical significance on adjustment for age, diabetes duration and sex. However it seems that plasma glucose is the most important determinant of the ethnic difference in risk since adjustment for glucose attenuates the relative risk to 1.1 .

\section{Discussion}

These data confirm the well-recognised importance of proteinuria as a predictor of renal failure in both Type I [9-12] and Type II diabetes [13, 14]. Light proteinuria was associated with a threefold increased risk of renal failure in patients with Type I diabetes and a twofold increase among those with Type II diabetes, adjusted for age. Although the salicylsulphonic acid test is no longer used it has been shown to have $100 \%$ sensitivity and $85 \%$ specificity for detecting urinary albumin at $30 \mathrm{mg} / \mathrm{l}$ concentration (compara- 
ble with the current definition of microalbuminuria) [15]. Those with heavy proteinuria at baseline were excluded because they were likely to have overt diabetic nephropathy.

These data also support the role of hyperglycaemia as a determinant of renal failure in Type II diabetic patients and thus the importance of maintaining optimal glycaemic control in this group. This finding is consistent with the many studies both cross-sectional [16] and prospective [17-20] showing that the degree of hyperglycaemia (measured as glycated $\mathrm{Hb}$ ) predicts either overt nephropathy or microalbuminuria in Type I and Type II diabetes. As a single fasting plasma glucose estimation was used, we were unable to assess the role of long-term glycaemic control in renal failure in Type I diabetes.

There has been considerable argument about the extent to which blood pressure has a causal role in diabetic nephropathy as distinct from being a consequence of it [21]. In other studies blood pressure was found to be higher in patients with microalbuminuria (before GFR decreases) than in those with normoalbuminuria, [10, 22, 23] but a Danish study suggested that blood pressure does not rise until microalbuminuria has been established for several years [24]. Studies showing that a parental history of hypertension is associated with nephropathy in the offspring $[25,26$, 44] suggest that, at least to some extent, elevations in blood pressure precede the onset of renal disease. In addition, some studies show that Type I diabetic patients who develop nephropathy have higher blood pressures in adolescence [27, 28]. In our data systolic blood pressure predicts renal failure even after adjusting for degrees of proteinuria at baseline but in Type I diabetic patients only. A systolic blood pressure one standard deviation above the mean was associated with a $50 \%$ higher risk of renal failure in Type I diabetes, after adjusting for other factors (Model 1, Table 3). Systolic blood pressure was not predictive of renal failure in Type II diabetic patients even before adjustment for baseline proteinuria. Similarly hypertension defined as a SBP of $140 \mathrm{mmHg}$ or more, or a DBP of $90 \mathrm{mmHg}$ or more, or use of antihypertensive medications was predictive of renal failure in Type I diabetic but not Type II diabetic patients. It should be noted that in our study as in others, casual blood pressure measurements were made rather than ambulatory measurements which could be more sensitive for the detection of hypertension.

In this study retinopathy was a predictor of renal failure even after adjusting for baseline proteinuria, systolic blood pressure, age, duration, and, in Type II diabetes, fasting blood glucose and triglycerides. The presence and severity of retinopathy could simply be a marker of the intensity of the microangiopathic process, driven by poor glycaemic control interacting with individual susceptibility [29]. This is reflected by the clustering of vascular complications. In the
EURODIAB Type I Complications Study there was an increase in albumin excretion rate with increasing systolic blood pressure only among those with retinopathy [30] but no such interaction was observed in this study.

In neither Type I nor Type II diabetes did we find any association between self-reported smoking (past or current) and renal failure either before or after adjusting for other possible risk factors. In contrast in the EURODIAB Type I Complications Study current smoking was associated with albuminuria [31], a finding consistent with most [32-35] but not all [36] other studies.

Low density lipoprotein (LDL) cholesterol and triglycerides increase with the onset of renal disease $[37,38]$, even at an early stage. A causal role for dyslipidaemia in nephropathy in Type I diabetes was suggested by the Pittsburgh Epidemiology of Diabetes Complications Study, which found that LDL and triglycerides predicted the development of albuminuria [17]. In Pima Indians, raised total plasma cholesterol predicted increased albumin excretion (data on triglycerides were not available) [18]. In the present study total cholesterol at baseline was not predictive of renal failure. In Type I diabetic patients the incidence of renal failure did increase with baseline triglyceride concentration (Table 2) but this association was not statistically significant. Among those with Type II diabetes, triglyceride concentrations were associated with renal failure independently of other risk factors including proteinuria at baseline. Indeed, in Type II diabetic patients triglycerides were more closely associated with renal failure than arterial pressure. There are several possible explanations for the association. Higher triglycerides could simply reflect poorer glycaemic control not fully accounted for by fasting plasma glucose (i.e. residual confounding). However, the WHO Multinational Study is large enough and of sufficiently long duration to detect associations that might have gone undetected in studies with less power and there are several biologically plausible mechanisms by which triglycerides could be truly predictive of renal disease. Raised triglycerides are associated with increased formation of small dense low density lipoproteins [39, 40] which in turn have increased susceptibility to oxidation and which could initiate endothelial damage and thus renal disease. Alternatively it is possible that the association between triglycerides and renal failure could have arisen because higher triglycerides are associated with higher concentrations of non-esterified fatty acids (NEFA). Non-esterified fatty acids have been shown to impair endothelial function, probably by inhibiting nitric oxide production [41, 42] and decreased nitric oxide production is associated with the development of diabetic nephropathy in diabetic spontaneously hypertensive rats [43]. Thus it is possible to speculate that high triglycerides could be a 
marker of impaired nitric oxide production with consequent renal damage.

The high renal failure rate in American Indians has been reported previously and is consistent with the higher incidence of renal failure in this group [4]. Our analysis shows that plasma glucose appears to be the most important determinant of this increased risk and suggest that triglycerides could also be involved (Table 4).

Our data have several limitations. It is possible that renal failure was unrecognised as a cause of some of the deaths reported. However, such underascertainment would reduce the power to detect associations with risk factors rather than creating spurious associations. We attempted to exclude those with established renal failure at baseline by excluding anyone with heavy proteinuria. It is possible, however, that some of the observed risk-factor associations reflect a secondary rise in risk factors in those with incipient renal disease at baseline. However, because the associations observed were all independent of even light proteinuria at baseline this is not likely.

A total of 645 patients who were alive at follow-up but whose renal status was not known were excluded from this analysis. The estimate of the incidence of renal failure could be either an underestimation (if all these 645 actually had renal failure) or an overestimation (if none of these 645 had renal failure). Slight overestimation is more likely, as the baseline risk factor profile of this group was more similar to those who did not subsequently develop renal failure than those who did.

In summary we have confirmed the role of proteinuria and retinopathy as markers of renal failure. The importance of hyperglycaemia in renal failure in Type II diabetes has been shown. Evidence has been found for a potentially important role for triglycerides as a predictor of renal failure in Type II diabetes.

\section{References}

1. Raine AEG (1997) Evolution worldwide of the treatment of patients with advanced diabetic nephropathy by renal replacement therapy. In: CE Mogensen (ed) The kidney and hypertension in diabetes mellitus, 3rd ed. Kluwer Academic publishers, Boston, pp. 473-480

2. Krolewski AS, Laffel LMB, Krolewski M, Quinn M, Warram JH (1995) Glycosylated hemoglobin and the risk of microalbuminuria in patients with insulin-dependent diabetes mellitus. N Engl J Med 332: 1251-1255

3. The Diabetes Control and Complications (DCCT) Research Group (1995) Effect of intensive therapy on the development and progression of diabetic nephropathy in the Diabetes Control and Complications Trial. Kidney Int 47: 1703-1720

4. Bennett PH, Lee ET, Lu M, Keen H, Fuller JH, The WHO Multinational Study Group (2001) Increased urinary albumin excretion and its associations in the WHO Multinational Study of Vascular Disease in Diabetes. Diabetologia 44 [Supp 2]: S 37-S 45
5. Lee ET, Keen H, Bennett PH, Fuller JH, Lu M, and the WHO Multinational Study Group (2001) Follow-up of the WHO Multinational Study of Vascular Disease in Diabetes: general description and morbidity. Diabetologia 44 [Supp 2]: S3-S13

6. Jarrett RJ, Keen H, Grabauskas V (1979) The WHO Multinational Study of Vascular Disease in Diabetes: 1 General description. Diabetes Care 2: 175-186

7. Diabetes Drafting Group (1985) Prevalence of small vessel and large vessel disease in diabetic patients from 14 centres. The WHO Multinational Study of Vascular Disease in Diabetics. Diabetologia 28: 615-640

8. West KM, Ahuja MMS, Bennett PH et al. (1983) The role of circulating glucose and triglyceride concentrations and their interactions with other "risk factors" as determinants of arterial disease in nine diabetic population samples from the WHO Multinational Study. Diabetes Care 6:361-369

9. Mogensen CE (1984) Microalbuminuria predicts clinical proteinuria and early mortality in maturity-onset diabetes. N Engl J Med 310: 356-360

10. Mogensen CE (1984) Predicting diabetic nephropathy in insulin-dependent patients. N Engl J Med 311: 89-93

11. Mathiesen ER, Oxenboll B, Johansen K, Svendsen PA, Deckert T (1984) Incipient nephropathy in Type I (insulin-dependent) diabetes. Diabetologia 26: 406-410

12. Viberti GC, Hill RD, Jarrett RJ, Argyropoulos A, Mahmud U, Keen H (1982) Microalbuminuria as a predictor of clinical nephropathy in insulin-dependent diabetes mellitus. Lancet i: 1430-1432

13. Nelson RG, Pettit DJ, Carraher MJ, Baird HR, Knowler WC (1988) Effect of proteinuria on mortality in Type II. Diabetes 37: 1499-1504

14. Neil A, Hawkins M, Potok M, Thorogood M, Cohen D, Mann J (1993) A prospective population-based study of microalbuminuria as a predictor of mortality in Type II. Diabetes Care 16: 996-1003

15. Watts GF, Hodgson B, Morris RW, Shaw KM, Polak A (1988) Side-room tests to screen for microalbuminuria in diabetes mellitus. Diabetic Med 5: 298-303

16. The EURODIAB Type I Complications Study Group (1994) Microvascular and acute complications in insulin dependent diabetes mellitus: the EURODIAB Type I Complications Study. Diabetologia 37: 278-285

17. Coonrod BA, Ellis D, Becker DJ et al. (1993) Predictors of microalbuminuria in individuals with Type I. Diabetes Care 16: 1376-1383

18. Nelson RG, Knowler WC, Pettitt DJ, Hanson RL, Bennett PH (1995) Incidence and determinants of elevated urinary albumin excretion in Pima Indians with Type II. Diabetes Care 18: 182-187

19. Kunzelman CL, Knowler WC, Pettitt DJ, Bennett PH (1989) Incidence of proteinuria in type 2 diabetes mellitus in the Pima Indians. Kidney Int 35: 681-687

20. Klein R, Klein BEK, Moss SE (1993) Incidence of gross proteinuria in older-onset diabetes - A population-based perspective. Diabetes 42: 381-389

21. Mogensen CE, Osterby R, Hansen KW, Damsgaard EM (1992) Blood pressure elevation versus abnormal albuminuria in the genesis and prediction of renal disease in diabetes. Diabetes Care 15: 1181-1204

22. Parving H, Smidt UM, Friisberg B, Bonnevie-Nielsen V, Andersen AR (1981) A prospective study of glomerular filtration rate and arterial blood pressure in insulin-dependent diabetics with diabetic nephropathy. Diabetologia 20: 457-461

23. Microalbuminuria Collaborative Study Group UK (1993) Risk factors for development of microalbuminuria in insu- 
lin dependent diabetic patients: a cohort study. BMJ 306: 1235-1239

24. Mathiesen E, Ronn B, Jensen T, Storm B, Deckert T (1990) Relationship between blood pressure and urinary albumin excretion in development of microalbuminuria. Diabetes 39: 245-249

25. Krolewski AS, Canessa M, Warram JH et al. (1988) Predisposition to hypertension and susceptibility to renal disease in insulin-dependent diabetes mellitus. N Engl J Med 318: 140-145

26. Viberti GC, Keen H, Wiseman MJ (1987) Raised arterial pressure in parents of proteinuric insulin dependent diabetics. BMJ 295: 515-517

27. Barzilay J, Warram JH, Bak M, Laffel LMB, Canessa M, Krolewski AS (1992) Predisposition to hypertension: risk factor for nephropathy and hypertension in Type I. Kidney Int 41: 723-730

28. Chase PH, Garg SK, Harris S, Hoops S, Marshall G (1990) High-normal blood pressure and early diabetic nephropathy. Arch Intern Med 150: 639-641

29. Jensen T (1991) Albuminuria-a marker of renal and generalized vascular disease in insulin-dependent diabetes mellitus. Dan Med Bull 38: 134-144

30. Stephenson JM, Fuller JH, Viberti G, Sjolie A, Navalesi R, The EURODIAB Type I Complications Study Group (1995) Blood pressure, retinopathy and urinary albumin excretion in Type I: the EURODIAB Type I complications study. Diabetologia 38: 599-603

31. Chaturvedi N, Stephenson JM, Fuller JH, The EURODIAB Type I Complications Study Group (1995) The relationship between smoking and microvascular complications in the EURODIAB Type I Complications Study. Diabetes Care 18: 785-792

32. Telmer S, Christiansen JS, Andersen AR, Nerup J, Deckert T (1984) Smoking habits and prevalence of clinical diabetic microangiopathy in insulin-dependent diabetics. Acta Med Scand 215: 63-68

33. Chase HP, Garg SK, Marshall G et al. (1991) Cigarette smoking increases the risk of albuminuria among subjects with type I diabetes. JAMA 265: 614-617
34. Sawicki P, Didjurgeit U, Muhlhauser I, Bender R, Heinemann L, Berger M (1994) Smoking is associated with progression of diabetic nephropathy. Diabetes Care 17: 126-131

35. Norden G, Nyberg G (1984) Smoking and Diabetic Nephropathy. Acta Med Scand 215: 257-261

36. Orchard TJ, Dorman JS, Maser RE et al. (1990) Factors associated with avoidance of severe complications after $25 \mathrm{yr}$ of Type I. Pittsburgh Epidemiology of Diabetes Complications Study I. Diabetes Care 13: 741-747

37. Vannini P, Ciavarella A, Flammini M et al. (1984) Lipid abnormalities in insulin-dependent diabetic patients with albuminuria. Diabetes Care 7: 151-154

38. Jensen T, Stender S, Deckert T (1988) Abnormalities in plasma concentrations of lipoproteins and fibrinogen in Type I (insulin-dependent) diabetic patients with increased urinary albumin excretion. Diabetologia 31: 142-145

39. Lahdenpera S, Groop P, Tilly-Kiesi M et al. (1994) LDL subclasses in Type I patients: relation to diabetic nephropathy. Diabetologia 37: 681-688

40. Taskinen MR, Lahdenpera S, Syvanne M (1996) New insights into lipid metabolism in non-insulin-dependent diabetes mellitus. Ann Med 28: 335-340

41. Steinberg HO, Paradisi G, Hook G, Crowder K, Cronin J, Baron AD (2000) Free fatty acid elevation impairs insulinmediated vasolidation and nitric oxide production. Diabetes 49: 1231-1238

42. Steinberg HO, Baron AD (1997) Insulin-dependent diabetes mellitus and nitrovasodilation. Important and complex interactions. Circulation 95: 560-561

43. Wessels J, Peake P, Pussell BA, Macdonald GJ (1997) Nitric oxide synthase inhibition in a spontaneously hypertensive rat model of diabetic nephropathy. Clin Exp Pharmacol Physiol 24: 451-453

44. Roglic G, Colhoun H, Stevens LK, Lemkes HH, Manes C, Fuller JH, and the EURODIAB Type I Complications Study Group (1998) Parental history of hypertension and parental history of diabetes and microvascular complications in insulin-dependent diabetes mellitus: the EURODIAB Type I Complications Study. Diabet Med 15: 418-426 